which also contained glioma-specific microRNAs and proangiogenic proteins. Glioblastomaproduced mRNA could be expressed in recipient cells; for example, human brain microvascular endothelial cells exposed to tumor microvesicles that contained a luciferase mRNA began to express the luciferase enzyme. Glioblastoma microvesicles also induced angiogenesis in endothelial cell cultures, and stimulated proliferation in a glioma cell line. Analysis of serum samples from patients with glioblastoma showed that the patients' EGFR variant III status could be determined from microvesicles carried in serum; EGFR variant III mRNA was found in 14 of 30 patients tested, but in none of 30 controls.

Analysis of glioblastoma microvesicles in serum could provide diagnostic information to monitor tumors and assist in the design of therapeutic strategies; also, the microvesicles themselves might have potential as delivery vehicles for anticancer agents.

Original article Skog J et al. (2008) Glioblastoma microvesicles transport RNA and proteins that promote tumour growth and provide diagnostic biomarkers. Nat Cell Biol 10: 1470-1476

\section{Uncoupling Src from the NMDA receptor treats chronic pain in rodents}

The $\mathrm{N}$-methyl-D-aspartate receptor (NMDAR) is centrally involved in the pathogenesis of chronic pain hypersensitivity. However, NMDARs are essential to important physiological processes, which limit the use of NMDAR blockers as therapy for chronic pain. Upregulation of the NMDAR is mediated by the Src tyrosine kinase in the NMDAR complex. A Canadian study has now shown that uncoupling Src from the NMDAR complex suppresses inflammatory and neuropathic pain hypersensitivity in rats or mice without inhibiting intrinsic NMDAR function.

Liu et al. identified a 10-residue peptide, comprising residues 40-49 of Src, which prevented binding of Src to the NMDAR. To permit cell permeability, this peptide was joined to the protein transduction domain of HIV Tat protein. Pretreatment of animals with the fused peptide reduced inflammatory pain responses to formalin. Administration of $\mathrm{Src}_{40-49}$ Tat to animals subjected to injection of complete Freund's adjuvant or peripheral nerve injury reduced the animals' hypersensitivity to thermal and mechanical pain. Injury-induced phosphorylation of the NMDAR NR2B subunit was reduced in the spinal cords of animals treated with $\mathrm{SrC}_{40-49}$ Tat compared with control mice. NMDAR-dependent learning was not affected by $\mathrm{SrC}_{40-49}$ Tat, as measured by fear-conditioning and taste-aversion tests.

The authors conclude that preventing the upregulation of NMDAR without affecting basal function has potential therapeutic value in the management of chronic pain, and possibly other NMDAR-dependent CNS disorders.

Original article Liu XJ et al. (2008) Treatment of

inflammatory and neuropathic pain by uncoupling Src from the NMDA receptor complex. Nat Med 14: 1325-1332

\section{Skin impedance is a potential marker for Fabry disease}

A US research group has developed a simple, easy-to-use method of screening for Fabry disease. The technique depends on the use of a dynamic dermal impedance monitor to measure differences in skin moisture between patients with Fabry disease and healthy control individuals.

Gupta et al. recruited 27 patients with Fabry disease (22 had received 3 years of enzyme replacement therapy and 5 were enzyme replacement therapy-naive) and 22 healthy controls. Skin impedance was measured with a dynamic dermal impedance monitor at 26 body locations; 110 readings (5 per second) were taken at each location. Four outcome measures were tested: the $100^{\text {th }}$ reading, the rate of change, the average of readings $60-110$ and the average of all 110 readings. The difference in skin impedance between patients and controls was statistically significant by all outcome measures for most skin sites; however, outcomes for patients on enzyme replacement therapy and enzyme replacement therapy-naive patients were not significantly different, possibly because the dynamic dermal impedance monitor measures basal rather than induced moisture.

Given the similarity between results for the different outcome measures, the authors recommend the $100^{\text {th }}$ reading as the most convenient to measure. The portability, ease of use and time-efficiency of the dynamic dermal impedance monitor means that this method 